\title{
PID tuning for position control of DC servo-motor using TLBO
}

\author{
S. Shrivastava ${ }^{1 *}$, V. P. Singh ${ }^{1}$, R. K. Dohare ${ }^{2}$, S. P. Singh ${ }^{1}$ and D. P. S. Chauhan ${ }^{1}$ \\ Department of Electrical Engineering, National Institute of Technology, Raipur, Chhattisgarh, India ${ }^{1}$ \\ Department of Chemical Electrical Engineering, National Institute of Technology, Jaipur, Rajasthan, India ${ }^{2}$
}

\section{(C)2017 ACCENTS}

\begin{abstract}
In this paper, an optimal proportional-integral-derivative (PID) controller is suggested for position control of DC servomotor system. The performance index considered is integral-square-error (ISE) of unit step response. The ISE is designed with the help of alpha and beta tables. The teaching-learning-based optimization (TLBO) algorithm is utilized for minimizing the performance index. The proposed results are compared with other existing well-known methods. It is found that the TLBO based tuning provided better results.
\end{abstract}

Keywords

ISE, Optimization, PID controller, Position control, Servo-motor, TLBO.

\section{Introduction}

Being simple and reliable, the proportional-integralderivative (PID) controllers are still used in various engineering applications. One of the most respected methods is Ziegler-Nichols (ZN) tuning rule [1]. Some other tuning rules include integral of square time weighted error (ISTE), Pessen integral of absolute error (PIAE), Kessler Landau Voda (KLV), some overshoot rule (SO-OV), no overshoot rule (NO-OV), Mantz-Tacconi Ziegler-Nichols (MT-ZN) and refined Ziegler-Nichols (R-ZN) [2].

Although rule based tuning criteria provide good dynamic response however they are not best suited for optimal control. Due to this various evolutionary and swarm intelligence based tuning methods have been proposed in literature. Luus-Jaakola optimization procedure for PID controller tuning [3], evolutionary computation based PID tuning [4], PID controller tuning based on particle swarm optimization [5], PID tuning using soft computing techniques [6], PID controller tuning based on genetic algorithm [7], etc. are some examples of evolutionary and swarm intelligence based tuning methods. This paper proposes swarm intelligence based tuning method which utilizes teachinglearning-based optimization (TLBO) algorithm. The TLBO is applied for optimizing various engineering problems [8-11].

*Author for correspondence
The advantages of TLBO algorithm are simplicity, efficiency and no algorithm-specific parameters. The design criterion considered in this work is integralsquare-error (ISE) of unit step response. The ISE is constructed in terms of alpha and beta parameters.

The brief layout of this paper is as follows. Section 2 describes the DC servo-motor and its model. Section 3 gives the structure of PID controller. The proposed method of tuning is discussed in section 4 . The teacher learner based optimization algorithm is detailed in section 5. Section 6 discusses the simulation parameters and results obtained. The conclusion is provided in section 7 .

\section{The servo-motor}

The block diagram shown in Figure 1 shows the closed loop position control of DC servo-motor.

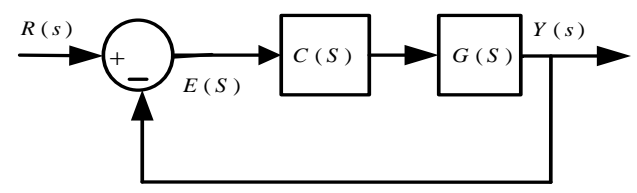

Figure 1 Closed loop position control of DC servomotor

The transfer function of plant i.e. DC servo-motor [12] is given as

$$
G(s)=\frac{K}{s\left(L_{a} s+R_{a}\right)\left(J_{0} s+B_{0}\right)+K K_{1} s}
$$


where, $K$ is electromotive force constant, $K_{1}$ is back EMF constant, $J_{0}$ is moment of inertia of motor, $B_{0}$ is viscous friction coefficient, $R_{a}$ is armature resistance and $L_{a}$ is armature inductance. The $C(s)$ in block diagram represents the controller.

\section{The controller}

The transfer function of proportional-integralderivative (PID) controller considered in this work is described as

$$
C(s)=K_{p}\left(1+\frac{1}{T_{i} s}+T_{d} s\right)
$$

where, $K_{p}, T_{i}$ and $T_{d}$ are, respectively, the proportional gain, integral time constant and derivative time constant. Other controller settings [2] due to Ziegler-Nichols (ZN) criterion, Pessen integral of absolute error (PIAE) criterion, some overshoot rule (SO-OV), no overshoot rule (NO-OV) and Mantz-Tacconi Ziegler-Nichols (MT-ZN) criterion are given in Table 1. The parameters $K_{u}$ and $T_{u}$ represent, respectively, the ultimate gain and corresponding period $T_{u}$.

Table 1 Controller parameters

\begin{tabular}{ccccc} 
S.N. & RULE & $\boldsymbol{K}_{\boldsymbol{p}}$ & $\boldsymbol{T}_{\boldsymbol{i}}$ & $\boldsymbol{T}_{\boldsymbol{d}}$ \\
\hline 1 & ZN & $0.6 K_{u}$ & $0.5 T_{u}$ & $0.125 T_{u}$ \\
2 & PIAE & $0.7 K_{u}$ & $0.4 T_{u}$ & $0.15 T_{u}$ \\
3 & SO-OV & $0.33 K_{u}$ & $0.5 T_{u}$ & $0.33 T_{u}$ \\
4 & NO-OV & $0.2 K_{u}$ & $0.5 T_{u}$ & $0.33 T_{u}$ \\
\cline { 3 - 5 }
\end{tabular}

$\begin{array}{lllll}5 & \text { MT-ZN } & 0.6 K_{u} & 0.5 T_{u} & 0.125 T_{u}\end{array}$

\section{The proposed tuning method}

The integral-square-error (ISE) of unit step input is minimized in this work. The error in s-domain for system given in Figure lcan be written as

$$
E(s)=R(s)-Y(s)=\frac{R(s)}{1+G(s) C(s)}
$$

where $R(s)$ and $Y(s)$ denotes respectively the desired input and actual output. The input is taken as

$$
R(s)=\frac{1}{s}
$$

In general, the error can be denotes as

$$
E(s)=\frac{b_{1} s^{n-1}+\cdots+b_{n}}{a_{0} s^{n}+a_{1} s^{n-1}+\cdots+a_{n}}
$$

where $n$ is the order of error $E(s)$.

The ISE

$$
J=\int_{t=0}^{t=\infty} e^{2}(t) d t
$$

for (5)in terms of alpha and beta parameters [13] is given as

$$
J=\frac{1}{2} \sum_{i=1}^{n} \frac{\beta_{i}^{2}}{\alpha_{i}}
$$

\begin{tabular}{|c|c|c|c|c|}
\hline & $\begin{array}{l}c_{\mathrm{o}}^{\mathrm{o}}=a_{\mathrm{o}} \\
c_{\mathrm{o}}^{1}=a_{1}\end{array}$ & $\begin{array}{l}c_{2}^{\mathrm{o}}=a_{2} \\
c_{2}^{1}=a_{3}\end{array}$ & $\begin{array}{l}c_{4}^{\mathrm{o}}=a_{4} \\
c_{4}^{1}=a_{5}\end{array}$ & $\begin{array}{c}c_{6}^{0}=a_{6} \\
\ldots\end{array}$ \\
\hline$\overline{\alpha_{1}}=c_{\mathrm{O}}^{\mathrm{O}} / c_{\mathrm{O}}^{1}$ & $c_{\mathrm{o}}^{2}=c_{2}^{\mathrm{o}}-\alpha_{1} c_{2}^{1}$ & $c_{2}^{2}=c_{4}^{\mathrm{O}}-\alpha_{1} c_{4}^{1}$ & $c_{4}^{2}=c_{6}^{\mathrm{O}}-\alpha_{1} c_{6}^{1}$ & $\cdots$ \\
\hline$\alpha_{2}=c_{0}^{1} / c_{0}^{2}$ & $c_{0}^{3}=c_{2}^{1}-\alpha_{2} c_{2}^{2}$ & $c_{2}^{3}=c_{4}^{1}-\alpha_{2} c_{4}^{2}$ & $c_{4}^{3}=c_{6}^{1}-\alpha_{2} c_{6}^{2}$ & $\ddots$ \\
\hline$\alpha_{3}=c_{0}^{2} / c_{0}^{3}$ & $c_{0}^{4}=c_{2}^{2}-\alpha_{3} c_{2}^{3}$ & $c_{2}^{4}=c_{4}^{2}-\alpha_{3} c_{4}^{3}$ & $\vdots$ & \\
\hline$\alpha_{4}=c_{0}^{3} / c_{0}^{4}$ & $c_{\mathrm{o}}^{5}=c_{2}^{3}-\alpha_{4} c_{2}^{4}$ & $\vdots$ & & \\
\hline $\begin{array}{c}\alpha_{5}=c_{\mathrm{O}}^{4} / c_{\mathrm{o}}^{5} \\
\vdots\end{array}$ & $\vdots$ & & & \\
\hline
\end{tabular}

The parameters $\alpha_{i}$ and $\beta_{i}$ for $i=1,2, \cdots, n$ are

\begin{tabular}{|c|c|c|c|c|}
\hline & $\begin{array}{l}d_{\mathrm{o}}^{1}=b_{1} \\
d_{\mathrm{o}}^{2}=b_{2}\end{array}$ & $\begin{array}{l}d_{2}^{1}=b_{3} \\
d_{2}^{2}=b_{4}\end{array}$ & $\begin{array}{l}d_{4}^{1}=b_{5} \\
d_{4}^{2}=b_{6}\end{array}$ & $\begin{array}{c}d_{6}^{1}=b_{7} \\
\ldots\end{array}$ \\
\hline$\beta_{1}=d_{\mathrm{o}}^{1} / c_{\mathrm{o}}^{1}$ & $d_{0}^{3}=d_{2}^{1}-\beta_{1} c_{2}^{1}$ & $d_{2}^{3}=d_{4}^{1}-\beta_{1} c_{4}^{1}$ & $d_{4}^{3}=d_{6}^{1}-\beta_{1} c_{6}^{1}$ & $\cdots$ \\
\hline$\beta_{2}=d_{0}^{2} / c_{0}^{2}$ & $d_{\mathrm{o}}^{4}=d_{2}^{2}-\beta_{2} c_{2}^{2}$ & $d_{2}^{4}=d_{4}^{2}-\beta_{2} c_{4}^{2}$ & $d_{4}^{4}=d_{6}^{2}-\beta_{2} c_{6}^{2}$ & $\because$ \\
\hline$\beta_{3}=d_{0}^{3} / c_{0}^{3}$ & $d_{\mathrm{o}}^{5}=d_{2}^{3}-\beta_{3} c_{2}^{3}$ & $d_{2}^{5}=d_{4}^{3}-\beta_{3} c_{4}^{3}$ & $\vdots$ & \\
\hline$\beta_{4}=d_{\mathrm{o}}^{4} / c_{\mathrm{O}}^{4}$ & $d_{\mathrm{o}}^{6}=d_{2}^{4}-\beta_{4} c_{2}^{4}$ & $\vdots$ & & \\
\hline $\begin{array}{c}\beta_{5}=d_{0}^{5} / c_{0}^{5} \\
\vdots\end{array}$ & $\vdots$ & & & \\
\hline
\end{tabular}
obtained from alpha table and beta table as given in Table 2 and Table 3.

Table 2 Alpha table

Table 3 Beta table 


\section{Teacher learner based optimization}

The teacher learner based optimization (TLBO) is recently proposed by Rao et al. [14]. It consists two phases: teacher phase and learner phase. In former phase, the teacher tries to improve the performance of class to his level. In latter phase, students interact to each other to improve their performance in various subjects offered to them.

Suppose, there is a total $R$ learners in the class and the subjects offered are $C$. The performance of $i$ th learner, $i=1,2, \cdots, R$, in $j$ th subject, $j=1,2, \cdots, C$ is $X_{i, j}$.

In teacher phase, teacher (i.e. best learner of the class) tries to improve the performance of students as:

$$
n e w X_{i, j}=X_{i, j}+r\left(X_{b e s t, j}-T_{f} \bar{X}_{i, j}\right)
$$

where newX $X_{i, j}$ is updated performance and $r$ is a random number such that $r=(0,1) . \quad X_{b e s t, j}$ and $\bar{X}_{i, j}$ represent respectively the performance of best student and average performance of the class. The parameter $T_{f}$ is known as teacher factor which is given as

$T_{f}=\{1,2\}$

At the end of teacher phase, new $X_{i, j}$ is updated if it is a better solution otherwise old solution is retained. The solution at the end of teacher becomes input to the learner phase. In learner phase, each learner interact to other as given by

$n e w X_{i, j}=X_{i, j}+r\left(X_{p, j}-X_{q, j}\right)$

where $p$ and $q$ are two randomly selected learners such that $p \neq q, f\left(X_{p, j}\right)<f\left(X_{q, j}\right)$ and $r=(0,1)$.

At the end of learner phase, the solution $n e w X_{i, j}$ is updated in $X$ if it is better otherwise $X_{i, j}$ is retained. This completes one iteration of TLBO algorithm. This process continues until desired criterion meets.

\section{Results and discussion}

The parameters of DC servo-motor [12] are given as $K=0.01, K_{1}=0.01, R_{a}=1, L_{a}=0.5, \quad J_{0}=0.01$ and $B_{0}=0.1$. For these values, (5) becomes

$E(s)=\frac{b_{1} s^{3}+b_{2} s^{2}+b_{3} s+b_{4}}{a_{0} s^{4}+a_{1} s^{3}+a_{2} s^{2}+a_{3} s+a_{4}}$

Where,

$a_{0}=T_{i} L_{a} J_{0}$

$$
\begin{aligned}
& a_{1}=T_{i}\left(L_{a} B_{0}+R_{a} J_{0}\right) \\
& a_{2}=T_{i}\left(B_{0} R_{a}+K K_{1}+K_{p} K T_{d}\right) \\
& a_{3}=T_{i} K K_{p} \\
& a_{4}=K K_{p} \\
& b_{1}=T_{i} L_{a} J_{0} \\
& b_{2}=T_{i}\left(L_{a} B_{0}+R_{a} J_{0}\right) \\
& b_{3}=T_{i}\left(B_{0} R_{a}+K K_{1}\right) \\
& b_{4}=0
\end{aligned}
$$

The alpha and beta tables (Table 2 and Table 3) for (11)becomes, respectively, Table 4 and Table 5.

Table 4 Alpha table

\begin{tabular}{l|lll} 
& $c_{0}^{0}=a_{0}$ & $c_{2}^{0}=a_{2}$ & $c_{4}^{0}=a_{4}$ \\
& $c_{0}^{1}=a_{1}$ & $c_{2}^{1}=a_{3}$ & $c_{4}^{1}=0$ \\
\hline$\alpha_{1}=c_{0}^{0} / c_{0}^{1}$ & $c_{0}^{2}=c_{2}^{0}-\alpha_{1} c_{2}^{1}$ & $c_{2}^{2}=c_{4}^{0}$ & \\
$\alpha_{2}=c_{0}^{1} / c_{0}^{2}$ & $c_{0}^{3}=c_{2}^{1}-\alpha_{2} c_{2}^{2}$ & $c_{2}^{3}=0$ & \\
$\alpha_{3}=c_{0}^{2} / c_{0}^{3}$ & $c_{0}^{4}=c_{2}^{2}$ & & \\
$\alpha_{4}=c_{0}^{3} / c_{0}^{4}$ & & &
\end{tabular}

Table 5 Beta table

$$
\begin{aligned}
& \begin{array}{l|ll}
d_{0}^{1}=b_{1} & d_{2}^{1}=b_{3} \\
d_{0}^{2}=b_{2} & d_{2}^{2}=0 \\
\hline \beta_{1}=d_{0}^{1} / c_{0}^{1} & d_{0}^{3}=d_{2}^{1}-\beta_{1} c_{2}^{1} &
\end{array} \\
& \beta_{2}=d_{0}^{2} / c_{0}^{2} \quad d_{0}^{4}=-\beta_{2} c_{2}^{2} \\
& \beta_{3}=d_{0}^{3} / c_{0}^{3} \\
& \beta_{4}=d_{0}^{4} / c_{0}^{4}
\end{aligned}
$$

The performance index, given by (7), takes place

$$
\begin{aligned}
& J=\frac{1}{2} \sum_{i=1}^{4} \frac{\beta_{i}^{2}}{\alpha_{i}}=\frac{1}{2}\left\{\frac{\beta_{1}^{2}}{\alpha_{1}}+\frac{\beta_{2}^{2}}{\alpha_{2}}+\frac{\beta_{3}^{2}}{\alpha_{3}}+\frac{\beta_{4}^{2}}{\alpha_{4}}\right\} \\
& J=\frac{1}{2}\left\{\begin{array}{l}
\frac{\left(b_{1} / a_{1}\right)^{2}}{a_{0} / a_{1}}+\frac{\left(b_{2} /\left(a_{2}-\alpha_{1} a_{3}\right)\right)^{2}}{a_{1} /\left(a_{2}-\alpha_{1} a_{3}\right)}+ \\
\frac{\left(\left(b_{3}-\beta_{1} a_{3}\right) /\left(a_{3}-\alpha_{2} a_{4}\right)\right)^{2}}{\left(a_{2}-\alpha_{1} a_{3}\right) /\left(a_{3}-\alpha_{2} a_{4}\right)}+ \\
\frac{\left(\left(b_{4}-\beta_{2} a_{4}\right) / a_{4}\right)^{2}}{\left(a_{3}-\alpha_{2} a_{4}\right) / a_{4}}
\end{array}\right\}
\end{aligned}
$$

The optimal results obtained by minimizing (22) using TLBO are provided in Table 6. Table 6 also tabulates the controller parameters obtained due to $\mathrm{ZN}, \mathrm{PIAE}, \mathrm{SO}-\mathrm{OV}, \mathrm{NO}-\mathrm{OV}$ and MT-ZN rules.

Figure 2 shows the unit step responses for proposed method and other existing settings. The settling time is provided in Table 7 . 
S. Shrivastavaet al.

Table 6 Controller parameters

\begin{tabular}{ccccc}
\hline S.N. & Rule & $\boldsymbol{K}_{\boldsymbol{p}}$ & $\boldsymbol{T}_{\boldsymbol{i}}$ & $\boldsymbol{T}_{\boldsymbol{d}}$ \\
\hline 1 & Proposed & 999.99 & 127.84 & 999.99 \\
2 & ZN & 72.07 & 0.70 & 0.17 \\
3 & PIAE & 84.08 & 0.56 & 0.21 \\
4 & SO-OV & 39.63 & 0.70 & 0.46 \\
5 & NO-OV & 24.02 & 0.70 & 0.46 \\
6 & MT-ZN & 72.07 & 0.70 & 0.17 \\
\hline
\end{tabular}

Table 7 Settling time

\begin{tabular}{ccc}
\hline S.N. & Rule & $\boldsymbol{T}_{\boldsymbol{s}}(\mathbf{S e c})$. \\
\hline 1 & Proposed & 0.65 \\
2 & ZN & 5.77 \\
3 & PIAE & 4.56 \\
4 & SO-OV & 5.59 \\
5 & NO-OV & 8.90 \\
6 & MT-ZN & 5.77 \\
\hline
\end{tabular}

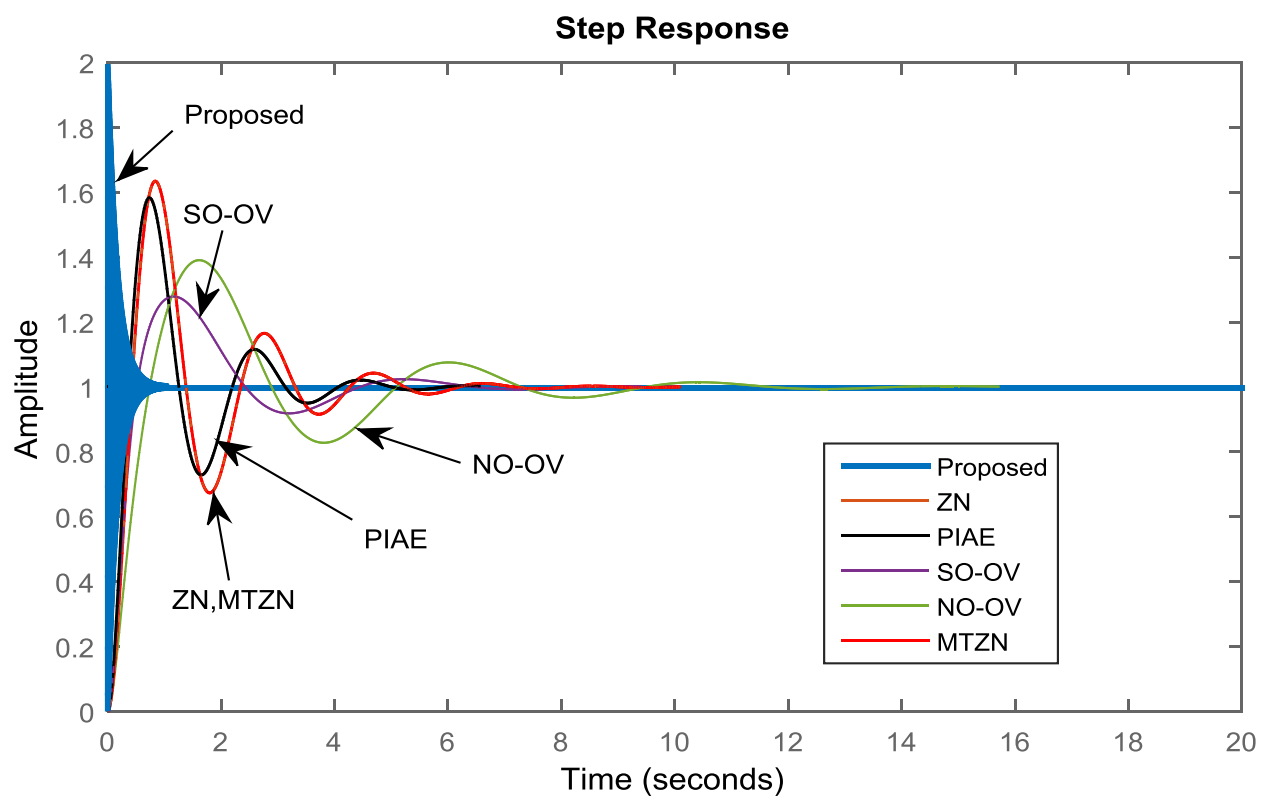

Figure 2 Step response of the system

From response plotted in Figure 2and settling time provided in Table 7, it is clear that the proposed method gives better results compared to other methods.

\section{Conclusion}

In this paper, an optimal PID controller for position control of DC servo-motor is proposed. For optimal settings, an ISE of unit step response is considered as design criterion. The ISE is obtained with the help of alpha and beta parameters. The teacher learner based optimization algorithm is employed for minimizing the ISE. The obtained controller parameters provide better performance in terms of qualitative and quantitative results.

\section{Acknowledgment}

None.

\section{Conflicts of interest}

The authors have no conflicts of interest to declare.

\section{References}

[1] Ziegler JG, Nichols NB. Optimum settings for automatic controllers. trans. ASME. 1942; 64(11):75965.

[2] McCormack AS, Godfrey KR. Rule-based autotuning based on frequency domain identification. IEEE Transactions on Control Systems Technology. 1998; 6(1):43-61.

[3] Rathore NS, Chauhan DP, Singh VP. Luus-Jaakola optimization procedure for PID controller tuning in reverse osmosis system. In proceedings 23rd IRF international conference, Chennai, India 2015 (pp. 12$5)$.

[4] Wadhwani S, Verma V. Evolutionary computation techniques based optimal PID controller tuning. International Journal of Engineering Trends and Technology. 2013; 4(6):2529-34.

[5] Rathore NS, Kundariya N, Narain A. PID controller tuning in reverse osmosis system based on particle swarm optimization. International Journal of Scientific and Research Publications. 2013; 3(6):1-5.

[6] Nagaraj B, Subha S, Rampriya B. Tuning algorithms for PID controller using soft computing techniques. International Journal of Computer Science and Network Security. 2008; 8(4):278-81. 
[7] Bindu R, Namboothiripad MK. Tuning of PID controller for DC servo motor using genetic algorithm. International Journal of Emerging Technology and Advanced Engineering. 2012; 2(3):310-4.

[8] Baykasoğlu A, Hamzadayi A, Köse SY. Testing the performance of teaching-learning based optimization (TLBO) algorithm on combinatorial problems: Flow shop and job shop scheduling cases. Information Sciences. 2014; 276:204-18.

[9] Singh M, Panigrahi BK, Abhyankar AR. Optimal coordination of directional over-current relays using teaching learning-based optimization (TLBO) algorithm. International Journal of Electrical Power \& Energy Systems. 2013; 50:33-41.

[10] Rao RV, Kalyankar VD. Parameters optimization of advanced machining processes using TLBO algorithm. EPPM, Singapore. 2011; 20:21-31.
[11] Zhile YA, Kang LI, Qun NI, Yusheng XU, Foley A. A self-learning TLBO based dynamic economic/environmental dispatch considering multiple plug-in electric vehicle loads. Journal of Modern Power Systems and Clean Energy. 2014; 2(4):298-307.

[12] K. Ogata, Modern control engineering: Prentice Hall PTR; 2001.

[13] Hutton M, Friedland B. Routh approximations for reducing order of linear, time-invariant systems. IEEE Transactions on Automatic Control. 1975; 20(3):32937.

[14] Rao RV, Savsani VJ, Vakharia DP. Teachinglearning-based optimization: a novel method for constrained mechanical design optimization problems. Computer-Aided Design. 2011; 43(3):303-15. 